\title{
System identification using an over-parametrized model class Improving the optimization algorithm
}

\author{
Tomas McKelvey and Anders Helmersson \\ Department of Electrical Engineering \\ Linkping University, S-581 83 Linkping, Sweden \\ WWW: http://wWw.control.isy.liu.se \\ Email: \{tomas, andersh\}@isy.liu.se
}

March 1999

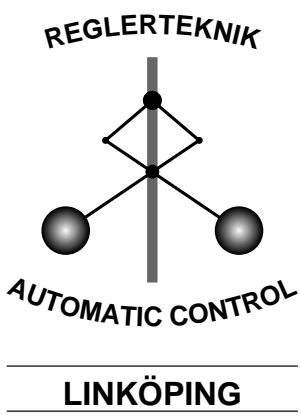

Report no.: LiTH-ISY-R-2136

Proc. 36th IEEE Conference on Decision and Control, San Diego, CA, 1997, pp 2984-2989

Technical reports from the Automatic Control group in Linkping are available by anonymous ftp at the address ftp.control.isy.liu.se. This report is contained in the pdf-file 2136.pdf. 


\title{
System identification using an over-parametrized model class - Improving the optimization algorithm ${ }^{1}$
}

\author{
Tomas McKelvey and Anders Helmersson \\ Dept. of Electrical Engineering, Linköping University \\ S-581 83 Linköping, Sweden, \\ Fax: +4613282622 \\ Email: tomas@isy.liu.se, andersh@isy.liu.se.
}

\begin{abstract}
The use of an over-parametrized state-space model for system identification has some clear advantages: A single model structure covers the entire class of multivariable systems up to a given order. The over-parametrization also leads to the possibility to choose a numerically stable parametrization. During the parametric optimization the gradient calculations constitute the main computational part of the algorithm. Consequently using more than the minimal number of parameters required slows down the algorithm. However, we show that for any chosen (over)parametrization it is possible to reduce the gradient calculations to the minimal amount by constructing the parameter subspace which is orthonormal to the tangent space of the manifold representing equivalent models.
\end{abstract}

\section{Introduction}

System identification deals with selecting a linear model from a model set using measured data. A set of models is most often described by a parametrized model structure. Hence, a parametrization is a mapping from the parameter space, a subset of $\mathbb{R}^{d}$, to the space of linear systems of given input-output configuration and state order. The best model in the set is determined by a parametric optimization of some criterion function.

We will here consider parametrizations and optimization of multivariable linear systems based on the statespace form

$$
\sigma x(t)=A x(t)+B u(t), \quad y(t)=C x(t)+D u(t)
$$

with $n$ states, $m$ inputs and $p$ outputs. For continuous time systems the operator $\sigma$ is the time differentiation

\footnotetext{
${ }^{1}$ This work was supported in part by the Swedish Research Council for Engineering Sciences (TFR), which is gratefully acknowledged.
}

$\frac{d}{d t}$ and for discrete time systems $\sigma$ is the forward time shift operator. A parametrization describes which matrix elements have fixed values and where the parameters enter into the system matrix elements.

Parametrization of multivariable linear systems is known as a difficult problem if a minimal parametrization is sought. In a minimal parametrization the mapping from parameter space to system space is injective, i.e. each system can be represented by at most one point in the parameter space. It is known [1] that the set of all possible input-output behaviors is a manifold of dimension $n(m+p)+p m$. The number of elements in the system matrices is however $n^{2}+n(m+p)+p m$. Consequently, in order to obtain an injective parametrization, $n^{2}$ degrees of freedom must be removed.

\subsection{Minimal Parametrizations}

In system identification injective parametrizations are known as being identifiable. If the parametrization is also bijective the parametrization can describe all systems of given order and input-output configuration. In this case each system corresponds to one and only one point in the parameter space. The use of an injective parametrization leads to a minimization problem that is well posed in the sense that the minimum will be unique in the parameter space if the data is informative. A caveat with a minimal parametrization is that the representation of a system close to the boundary of the set of reachable systems is numerically sensitive. Also models based on polynomial type parametrizations are highly unreliable when the order is high.

If the parametrization is bijective it is sufficient, for each model order, to only consider one parametrization and consequently only have to run one optimization. For single input multiple output systems or multi input single output systems the standard controllable canonical form and the observable canonical form are examples of bijective parametrizations [6].

A classical result tells us that if both the input and output dimensions are larger than one, a bijective 
parametrization does not exist $[8,4]$. Partially overlapping, injective parametrizations, based on the companion matrix form, has been used for multivariable system identification [13]. In this type of parametrizations the number of parameters, which is the minimal number, is $d_{\text {min }}=n(m+p)+m p$ where $n$ is the state dimension, $m$ the input dimension and $p$ the output dimension. For the observable form there exist $\left(\begin{array}{l}n-1 \\ p-1\end{array}\right)$ different structures and for the controllable form $\left(\begin{array}{l}n-1 \\ m-1\end{array}\right)$ structures. The model sets spanned by these parametrizations are partially overlapping and the union of all these equals the set of all dynamical systems of fixed input-output dimensions and fixed state order. The obvious drawback with such a parametrization is that several, or all, parametrizations should be considered when identifying a model from data without any prior structural knowledge. Even though one structure is capable of describing almost the entire set of systems it is likely that such a parametrization is numerically less favorable for a much larger class near the border of the set.

Based on the balanced realization a minimal (injective) parametrization has also been developed [12] and studied in an identification context in [9]. They argue that the balanced realization in general offer a more well conditioned parametrization as compared with the controllable-observable forms.

A second approach is to start with a structural identification revealing the internal structure and the appropriate parametrization. In a second step the parametric identification is then performed using the selected parametrization [4].

\subsection{Non-minimal Parametrizations}

If we drop the requirement of injectiveness of the parametrization and instead consider surjective parametrizations, the full parametrizations is one trivial choice. In this parametrization all matrix elements of the state space matrices $(A, B, C, D)$ are parameters. In an identification context such a parametrization was proposed in [10]. An obvious drawback with the full parametrization is the large number of parameters which have to be estimated:

$$
d_{\text {full }}=n^{2}+n(m+p)+m p .
$$

In comparison with the minimal parametrization the full parametrization has $n^{2}$ extra, redundant parameters. When using a parametrization with redundant parameters the minimum of the criterion function will not be unique in the parameter space. This leads to a singular Hessian of the criterion function. However, trivial modifications to standard Newton-type optimization algorithms ensure that a minimum is reached in a numerically stable stable way.

A surjective parametrization based on a tridiagonal structure of the $A$ matrix has recently been proposed
[11]. Although this is still an over-parametrized model structure it has only $2 n-1$ extra parameters. Since it is based on a tridiagonal $A$ matrix the poles (eigenvalues of $A$ ) are represented in a numerically stable way as opposed to the polynomial-type forms, e.g. observable and controllable canonical forms [14].

\subsection{Dynamically reducing the parameter space} During each step in a parametric optimization using a Gauss-Newton type algorithm [2] the gradient of the criterion function with respect to the parameters is evaluated. For most problems this is what constitutes the main computational load of the identification.

Consider the full parametrization when all matrix elements are considered as parameters. Take a point in this $n^{2}+n(m+p)+m p$ dimensional space. Since the manifold of systems has dimension $n(m+p)+m p$ there exist a manifold in the parameter space of dimension $n^{2}$ which represent all dynamical systems with identical input-output properties. We call these an equivalence class. When determining the gradient of the criterion function we know that it is zero for $n^{2}$ directions representing the tangent space of the manifold of the equivalence class. Consequently if we can determine this tangent space it is only necessary to evaluate the gradient in the directions perpendicular to the tangent space, which is of the minimal dimension $n(m+p)+m p$. A similar approach can also be found in [15] for identification using a LFT-type parametrisation.

A different approach with the same goal of minimizing the numerical burden of calculating the gradient is reported in [5]. They note that the filters, which are of the order of the number of parameters, representing the gradient function is not controllable and can be reduced. A drawback with their method is that the determination of controllability and transformation to the minimal system can be a numerically unstable process if the model orders are high.

In section 2 we will show how the tangent space of the equivalence class is easily determined and how to modify a standard Gauss-Newton algorithm to incorporate this information. In section 3 we will present some preliminary results from Monte-Carlo simulations comparing the proposed technique with the classical approach.

\section{A modified NLLS optimization algorithm}

Many identification problems can be cast as the minimization of a sum of squared errors [7]

$$
\hat{\theta}=\min _{\theta} V_{N}(\theta), \quad V_{N}(\theta)=\sum_{k=1}^{N} e(k, \theta)^{2}=\|E(\theta)\|^{2}
$$


where $E(\theta)$ is the column vector of the residuals $e(k, \theta)$. This problem is normally known as non-linear leastsquares (NLLS). If the residuals are a linear function of the parameters the problem simplifies to a classical least-squares (LS) problem which has an analytical solution. A first order expansion of $E(\theta)$ around $\theta$

$$
E(\theta+\delta \theta)=E(\theta)+\Psi(\theta) \delta \theta+\text { higher order terms }
$$

where $\Psi(\theta)=\left.\frac{d}{d \alpha} E(\alpha)\right|_{\alpha=\theta}$ is the Jacobian of the error vector, approximates the NLLS problem with a LS problem. The Jacobian matrix can easily be determined by the finite difference method. Let $e_{k}$ denote the $k$-th unit vector and let $X_{(:, k)}$ denote the $k$ th column of the matrix $X$. Consequently $e_{k}=I_{(:, k)}$ where $I$ is the identity matrix. The gradient of $E(\theta)$ with respect to $\theta_{k}$, the $k$ th element of $\theta$ is given by

$$
\Psi(\theta)_{(:, k)}=\frac{d}{d \theta_{k}} E(\theta)=\lim _{\gamma \rightarrow 0} \frac{E\left(\theta+e_{k} \gamma\right)-E(\theta)}{\gamma}
$$

A finite difference approximation is obtained by letting $\gamma$ above be a small fixed value. In general the finite difference method only gives an approximation of the true gradient. However, for some prediction error identification problems, such us the output error (OE) problem, it is easy to show that the finite difference method yields the exact gradient for any $\gamma>0$.

By using the first order expansion the criterion becomes

$$
V(\theta+\delta \theta) \approx\|E(\theta)+\Psi(\theta) \delta \theta\|^{2}
$$

which is a quadratic function of $\delta \theta$. The Gauss-Newton (GN) algorithm is based on a successive minimization of the first order approximation (2).

\section{Algorithm 1 (GN)}

1. Determine $\Psi(\theta)$ according to (1)

2. Determine the Gauss-Newton step $\delta \theta$ as the minimizer of

$$
\min _{\delta \theta}\|E(\theta)+\Psi(\theta) \delta \theta\|^{2}
$$

3. Perform a line search along the Gauss-Newton direction $\delta \theta$

$$
\min _{\mu} V(\theta+\mu \delta \theta)
$$

If no improvement is found then stop otherwise let $\theta:=\theta+\mu \delta \theta$ and go to step 1 .

When solving the least-squares problem (3) in step 2 a numerically stable algorithm should be used, e.g. using a QR-factorization or the singular value decomposition, see [3]. The quality of the search direction is tightly connected to the condition number of the gradient matrix $\Psi(\theta)$. The line search in step 3 can be done in many different ways. A simple and fairly robust solution is to start with $\mu=1$ and then bisect until a lower value of the criterion is found. The presentation here is only intended as an introduction to the technique. For further information see [2].
2.1 Tangent plane of the equivalence class

The transfer function of a linear system

$$
G(\sigma)=D+C(\sigma I-A)^{-1} B
$$

is invariant under similarity transformations, i.e. a change of basis of the state variables. A state space realization $(\tilde{A}, \tilde{B}, \tilde{C}, \tilde{D})$ is similar to $(A, B, C, D)$ if there exists a non-singular $T$ such that

$$
\left[\begin{array}{c|c}
\tilde{A} & \tilde{B} \\
\hline \tilde{C} & \tilde{D}
\end{array}\right]=\left[\begin{array}{c|c}
T A T^{-1} & T B \\
\hline C T^{-1} & D
\end{array}\right]
$$

If two realizations are similar their corresponding transfer functions are equal. The $n^{2}$ elements of the transformation matrix $T$ parametrize the equivalence class. In order to find the tangent plane of the manifold we only need to find the local linear transformation around $T=I$. Consider a small perturbation $T=I+\Delta T$. Since $(I+\Delta T)^{-1}=I-\Delta T$ we obtain, as a first order approximation,

$$
\left[\begin{array}{c|c}
\tilde{A} & \tilde{B} \\
\hline \tilde{C} & \tilde{D}
\end{array}\right]=\left[\begin{array}{c|c}
A & B \\
\hline C & D
\end{array}\right]+\left[\begin{array}{c|c}
\Delta T A-A \Delta T & \Delta T B \\
\hline-C \Delta T & D
\end{array}\right]
$$

If a full parametrization is used the parameter vectors can be constructed as

$$
\theta=\left[\begin{array}{c}
\operatorname{vec}(A) \\
\operatorname{vec}(B) \\
\operatorname{vec}(C) \\
\operatorname{vec}(D)
\end{array}\right] \quad \text { and } \quad \tilde{\theta}=\left[\begin{array}{c}
\operatorname{vec}(\tilde{A}) \\
\operatorname{vec}(\tilde{B}) \\
\operatorname{vec}(\tilde{C}) \\
\operatorname{vec}(\tilde{D})
\end{array}\right]
$$

where $\operatorname{vec}(\cdot)$ is the vectorization operator that forms a vector from a matrix by stacking the columns on top of each other. By use of the Kronecker product and the relation $\operatorname{vec}(A B C)=C^{T} \otimes A \operatorname{vec}(B)$, we can rewrite (5) as

$$
\tilde{\theta}=\theta+Q \operatorname{vec}(\Delta T)
$$

where

$$
Q=\left[\begin{array}{c}
A^{T} \otimes I_{n}-I_{n} \otimes A \\
B^{T} \otimes I_{n} \\
-I_{n} \otimes C \\
0
\end{array}\right]
$$

where $I_{n}$ denotes the $n \times n$ identity matrix. The columns of the indicated matrix $Q$ consequently spans the tangent plane of the manifold of the equivalence class at the point $\theta$. Let us summarize the result

Lemma 1 Consider a fully parametrized state-space model $(A, B, C, D)$ where all elements are parameters from a space $\mathbb{R}^{n(n+m+p)+m p}$. Let the manifold of all systems similar to $(A, B, C, D)$ be denoted by $M$. Then the tangent plane of $M$ at $(A, B, C, D)$ is spanned by the columns of $Q$ in (6). 
When deriving the gradient of the criterion function there is no point to use the $n^{2}$ directions spanned by the columns of $Q$ since we know that the gradient is zero in those directions. Instead we propose to use the orthonormal complement to $Q$ denoted by $Q^{\perp}$ as a basis for the determination of the non-zero gradient. A convienient way of obtaining $Q^{\perp}$ is by the singular value decomposition (SVD). Let the SVD of $Q$ be given by

$$
Q=\left[\begin{array}{ll}
U_{1} & U_{2}
\end{array}\right]\left[\begin{array}{cc}
\Sigma_{1} & 0 \\
0 & 0
\end{array}\right]\left[\begin{array}{l}
V_{1}^{T} \\
V_{2}^{T}
\end{array}\right]
$$

where $\left[\begin{array}{ll}U_{1} & U_{2}\end{array}\right]$ and $\left[V_{1} V_{2}\right]$ are square and unitary matrices and $\Sigma_{1}>0$. Then we obtain

$$
Q^{\perp}=U_{2} .
$$

Since we assume $\Sigma_{1}>0$ this implies that the number of columns in $U_{1}$ equals the rank of $Q$. Denote by $d_{\perp}$ the number of columns in $Q^{\perp}$. Then we have

$$
d_{\perp}=n^{2}+n(m+p)+m p-\operatorname{rank}(Q)
$$

In the normal case $\operatorname{rank}(Q)=n^{2}$ and it suffices to use the minimal number of search directions when determining the gradient. If $Q$ is rank deficient $Q^{\perp}$ is extended with a number of extra columns equal to the rank deficiency of $Q$. Necessary and sufficient conditions for $Q$ to have full rank remains to be investigated.

\subsection{An algorithm with search dimension reduc- tion}

With the knowledge of the subspace orthonormal to the tangent plane of the equivalence class we can modify the way we derive the Jacobian matrix. Consider again a linearization of the prediction errors

$$
E(\theta+\delta \theta) \approx E(\theta)+\Psi(\theta) \delta \theta
$$

By restricting the parameter update to directions orthogonal to the equivalence class we can write the perturbation as a function of a vector $\tilde{\delta \theta} \in \mathbb{R}^{d_{\perp}}$

$$
\delta \theta(\tilde{\delta \theta})=Q^{\perp} \tilde{\delta \theta}
$$

where $Q^{\perp}$ is the orthogonal complement to $Q$, see (6) and (7). With this restriction the linearization becomes

$$
E(\theta+\delta \theta(\tilde{\delta \theta})) \approx E(\theta)+\tilde{\Psi}(\theta) \tilde{\delta \theta}
$$

where

$$
\tilde{\Psi}(\theta)=\Psi(\theta) Q^{\perp}
$$

The key idea is that we can determine $\tilde{\Psi}(\theta)$ without first forming $\Psi(\theta)$. This is done by finite difference calculation using the columns of $Q^{\perp}$ as unit vectors. More specifically the gradient with respect to $\tilde{\delta} \theta_{k}$ is given by

$$
\begin{aligned}
& \tilde{\Psi}(\theta)_{(:, k)}=\frac{d}{d \tilde{\delta} \theta_{k}} E(\theta+\delta \theta(\tilde{\delta \theta})) \\
&=\lim _{\gamma \rightarrow 0} \frac{E\left(\theta+Q_{(:, k)}^{\perp} \gamma\right)+E(\theta)}{\gamma}
\end{aligned}
$$

Based on these observations we can modify the basic Gauss-Newton algorithm to only consider parameter updates in the directions orthogonal to the tangent plane of the equivalence class. By doing so we reduce the number of gradient calculations by $\operatorname{rank}(Q)$ (normally $\left.n^{2}\right)$ since the reduced gradient matrix $\tilde{\Psi}(\theta)$ has $\operatorname{rank}(Q)$ less columns than $\Psi(\theta)$.

\section{Algorithm 2 (GNSDR)}

1. Determine $Q^{\perp}$ using (7) and (6)

2. Determine $\tilde{\Psi}(\theta)$ according to (9)

3. Determine the minimizer of

$$
\min _{\tilde{\delta} \theta}\|E(\theta)+\tilde{\Psi}(\theta) \tilde{\delta \theta}\|^{2}
$$

The Gauss-Newton search direction with reduced dimension is given by

$$
\delta \theta=Q^{\perp} \tilde{\delta \theta}
$$

4. Perform a line search along the Gauss-Newton direction $\delta \theta$

$$
\min _{\mu} V(\theta+\mu \delta \theta)
$$

If no improvement found then stop otherwise let $\theta:=\theta+\mu \delta \theta$ and go to step 1 .

By using the presented GNSDR algorithm we can use the full parametrization with all its favorable properties without increasing the numerical burden as compared to using an injective parametrization with a minimal number of parameters. In the next section we investigate the numerical properties of the proposed algorithm by considering a simple identification problem.

\section{A numerical comparison}

In this section we use a small identification problem to illustrate the numerical properties of the proposed GNSDR algorithm. Particularly we will see how the condition number of the Jacobian matrix in the GaussNewton algorithm influences the probability to converge to the global minimum. An almost identical example was used in [11] to evaluate the properties of the tridiagonal parametrization. We will identify a system from a noise free impulse response using two different schemes. 
- The full parametrization and the proposed Algorithm 2 with reduced search dimension (GNSDR).

- The parametrization based on the controllable canonical form and the standard Gauss-Newton Algorithm 1 (GN).

We will study the condition number of the gradients $\Psi(\theta)$ for Algorithm 1 and $\tilde{\Psi}(\theta)$ for Algorithm 2 during the optimizations. We define the condition number of a matrix as

$$
\operatorname{cond}(X)=\frac{\max _{k} \sigma_{k}}{\min _{k} \sigma_{k}}
$$

where $\sigma_{k}$ are the singular values of the matrix $X$. Recall that a large condition number leads to a numerically unstable determination of the Gauss-Newton direction when solving (2).

The systems we will identify have the transfer function

$$
G_{0}(s)=\sum_{k=1}^{M} \frac{1}{s^{2}+2 \zeta_{k} \omega_{k} s+\omega_{k}^{2}}
$$

with natural frequency $\omega_{k}=1+4(k-1) /(M-1)$ and damping ratio $\zeta_{k}=0.1$. Denote by $g_{0}(t)$ the impulse response of $G_{0}$. The data used for the identification are 200 samples of the noise-free impulse response $g_{0}(t)$ of the system sampled with a period time $T=0.1$. Six different models are considered with $M=1, \ldots, 6$ representing model orders ranging from $2,4, \ldots, 12$.

The parametric optimization minimizes the nonlinearleast squares criterion function

$$
V(\theta)=\sum_{k=1}^{N}\left|g_{0}(k T)-\hat{g}(k, \theta)\right|^{2}
$$

In $(12), \hat{g}(k, \theta)$ is the impulse response of a parametrized discrete time system. Since the data is noise-free the criterion $V(\theta)$ should be zero at a minimum. The minimization is terminated when norm of the Gauss-Newton step falls below $10^{-5}$ or if the relative improvement of the criterion function is less than $10^{-4}$ or if more than 2000 criterion evaluations has occured. The optimization is considered successful if the algorithm terminates with $V(\theta)<10^{-8}$.

The optimization algorithm is initiated with a parameter value derived from a perturbed system. The perturbation is constructed by forming the sample and hold equivalence of $G_{0}(s)$ in balanced form and perturbing all matrix elements with zero mean Gaussian random noise with variance $10^{-4}$. The perturbed balanced form is used as the initial point for the full parametrization. For the minimal parametrization the initial point

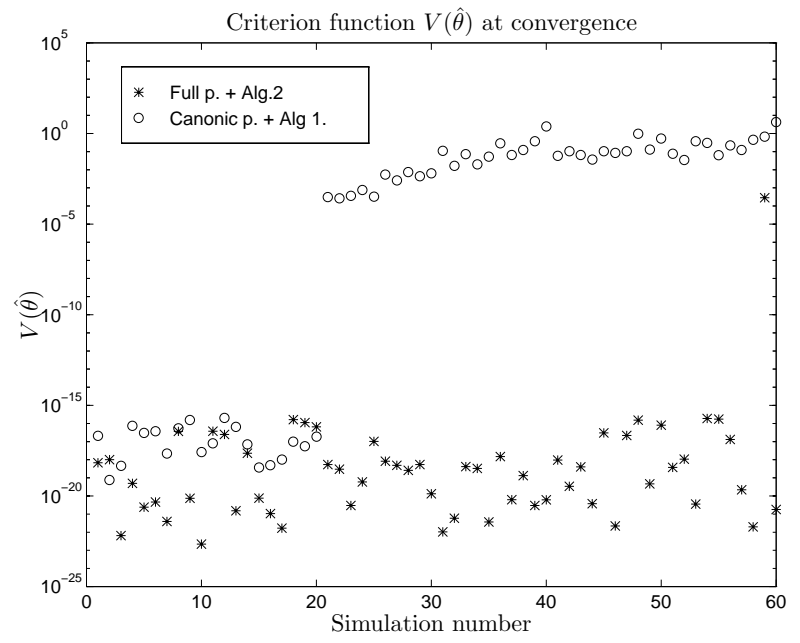

Figure 1: Results from Monte-Carlo simulations. The criterion function $V(\hat{\theta})$ at convergence. Note that for simulations 21-60 representing model orders $6,8,10$ and 12 the canonical parametrization failed to converge to the true system.

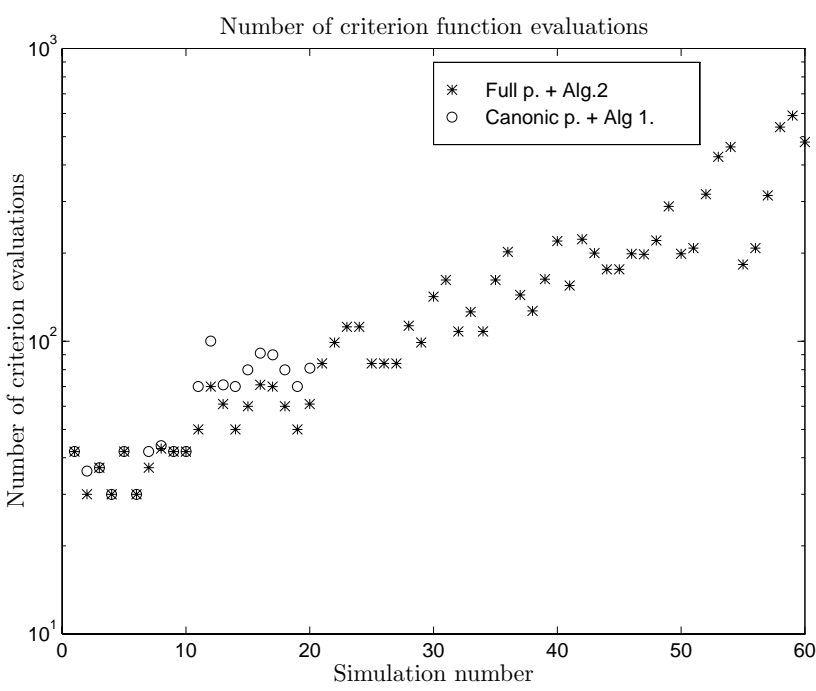

Figure 2: Results from Monte-Carlo simulations. Number of criterion function evaluations during the optimization including the calculation of the gradient by the finite difference method. Only simulations 1-20 are shown for the canonical parametrization since the rest failed to converge to the true system.

is converted from the balanced form to the controllable canonical form.

Figures 1 to 3 report the results of the Monte Carlo simulations. For each experimental setup, ten optimizations are performed using different initial parameters. Simulations 1-10 have a model order of 2, 11-20 model order $4 \ldots$ simulations $51-60$ have a model order of 12 . 


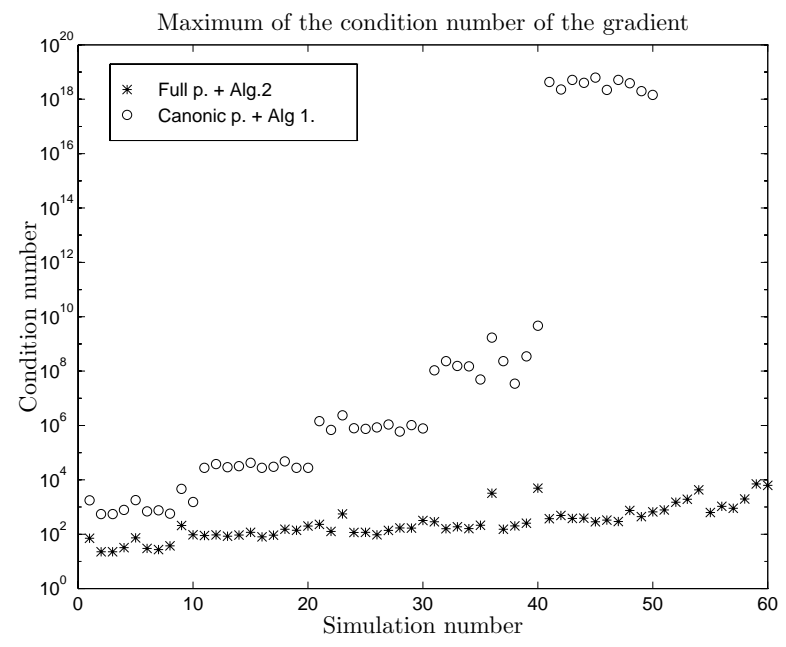

Figure 3: Results from Monte-Carlo simulations. Maximal value of the condition number of the gradient $(\Psi(\theta)$ and $\tilde{\Psi}(\theta))$ during the optimization.

From the results it is clear that the full parametrization together with Algorithm 2 (GNSDR) leads to a higher probability to reach the global minimum when the model complexity increases. By studying Figure 3 , which depicts the maximal condition number for each optimization, we notice that the condition numbers increases to large levels quickly for the canonical parametrization as the model complexity increases. This leads to a very unstable determination of the Gauss-Newton search direction $\delta \theta$ and convergence to local minima.

\section{Conclusions}

The use of an over-parametrized state-space model for system identification has some clear advantages; a single model structure covers the entire class of multivariable systems up to a given order. The overparametrization also leads to the possibility to choose a numerically stable parametrization. During the parametric optimization the gradient calculations constitute the main computational load of the algorithm. Consequently using more than the minimal number of parameters required slows down the algorithm. However, we have shown that for a full-parametrization it is possible to reduce the gradient calculations to the minimal amount by constructing the parameter subspace which is orthonormal to the tangent space of the manifold representing equivalent models. MonteCarlo simulations of one identification example illustrates that the proposed method show a higher probability to converge to the global minimum than the classical approach. The simulations also show that the condition number of the associated least-squares problem is significantly reduced for the proposed technique.

\section{References}

[1] J. M. C. Clark. The consistent selection of parametrizations in system identification. In Proc. Joint Automatic Control Conference (JACC), pages 576-580, Purdue University, Lafayette, Indiana, 1976.

[2] J. E. Dennis and R. B. Schnabel. Numerical Methods for Unconstrained Optimization and Nonlinear Equations. Prentice-Hall, Englewood Cliffs, New Jersey, 1983.

[3] G. H. Golub and C. F. Van Loan. Matrix Computations. The Johns Hopkins University Press, Baltimore, Maryland, second edition, 1989.

[4] R. Guidorzi. Canonical structures in the identification of multivariable systems. Automatica, 11:361374, 1975.

[5] N. K. Gupta and R. K. Mehra. Computational aspects of maximum likelihood estimation and reduction in sensitivity function calculations. IEEE Trans. on Automatic Control, 19(6):774-783, December 1974.

[6] T. Kailath. Linear Systems. Prentice-Hall, Englewood Cliffs, New Jersey, 1980.

[7] L. Ljung. System Identification: Theory for the User. Prentice-Hall, Englewood Cliffs, New Jersey, 1987.

[8] D. G. Luenberger. Canonical forms for linear multivariable systems. IEEE Trans. on Automatic Control, AC-12:290, 1967.

[9] J. M. Maciejowski and C. T. Chou. Applications of estimation using balanced parametrisations. In Proc. Third European Control Conference, volume 2, pages 1348-1351, Rome, Italy, 1995.

[10] T. McKelvey. Fully parametrized state-space models in system identification. In Proc. of the 10th IFAC Symposium on System Identification, volume 2, pages 373-378, Copenhagen, Denmark, July 1994.

[11] T. McKelvey and A. Helmersson. State-space parametrizations of multivariable linear systems using tridiagonal matrix forms. In Proc. 35th IEEE Conference on Decision and Control, pages 3654-3659, Kobe, Japan, December 1996.

[12] R. J. Ober. Balanced realizations: Canonical form, parametrization, model reduction. Int. J. Control, 46(2):643-670, 1987.

[13] A. J. M. van Overbeek and L. Ljung. On-line structure selection for multivariable state space models. Automatica, 18(5):529-543, 1982.

[14] J. H. Wilkinson. The Algebraic Eigenvalue Problem. Oxford University Press, London, 1965.

[15] G. Wolodkin, S. Rangan, and K. Poolla. An LFT approach to parameter estimation. In Preprints to 11th IFAC Symposium on System Identification, volume 1, pages 87-92, Kitakyushu, Japan, July 1997. 